\title{
Einige geometrische Sätze.
}

In Folge des Lehrsatzes 11. Band 6. Heft 2. S. 213.

(Von Herrn L. J. Magnus zu Berlin.)

\section{1.}

Es. sei $y=\varphi(x)$ die Gleichung irgend einer Curve, an welcher sich zwei Tangenten so bewegen, dafs sie immer denselben gegebenen Winkel mit einander bilden.

Nennt man die Coordinaten der Berührungspuncte jener Tangenten $\alpha, \beta$ und $\alpha^{\prime}, \beta^{\prime}$; und die trigonometrische Tangente des gegebenen Winkels $a$, so hat man, aufser den Gleichungen $\beta=\varphi(\alpha)$ und $\beta^{\prime}=\varphi\left(\alpha^{\prime}\right)$, zur Bestimmung des Ortes des Durchschnittspuncts der Tangenten, dessen Coordinaten $x, y$ heilsen mögen:

$$
\begin{aligned}
\text { 1. }(y-\beta) & =\frac{\partial \beta}{\partial \alpha}(x-\alpha), \\
\text { 2. }\left(y-\beta^{\prime}\right) & =\frac{\partial \beta^{\prime}}{\partial \alpha^{\prime}}\left(x-\alpha^{\prime}\right), \\
\text { 3. } \frac{\partial \beta}{\partial \alpha}-\frac{\partial \beta^{\prime}}{\partial \alpha^{\prime}} & =a\left(1+\frac{\partial \beta}{\partial \alpha} \frac{\partial \beta^{\prime}}{\partial \alpha^{\prime}}\right) .
\end{aligned}
$$

Eliminirt man zwischen diesen Gleichungen die Gröfsen $\frac{\partial \beta}{\partial \alpha}$ und $\frac{\partial \beta^{\prime}}{\partial \alpha^{\prime}}$, so kommt

4. $a\left[(y-\beta)\left(y-\beta^{\prime}\right)+(x-\alpha)\left(x-\alpha^{\prime}\right]+\left(y-\beta^{\prime}\right)(x-\alpha)-(y-\beta)\left(x-\alpha^{\prime}\right)=0\right.$, welche Gleichung, wenn man nur $x$ und $y$ als verïnderlich ansieht, einen Kreis ausdrückt, der durch die Puncte $\alpha, \beta$ und $\alpha^{\prime}, \beta^{\prime}$ geht. Differentiirt man diese Gleichung, indem man auch $\alpha, \beta$ und $\alpha^{\prime}, \beta^{\prime}$ als veränderlich betrachtet, so erhält man:

5. $\left[2 a y-a\left(\beta+\beta^{\prime}\right)+\alpha^{\prime}-\alpha\right] \partial y+\left[2 c x-a\left(\alpha+\alpha^{\prime}\right)+\beta-\beta^{\prime}\right] \partial x$

$$
\begin{aligned}
& +\left[y-\beta-a(x-\alpha)-[x-\alpha+a(y-\beta)] \frac{\partial \beta^{\prime}}{\partial \alpha^{\prime}}\right] \partial \alpha^{\prime} \\
& -\left[y-\beta^{\prime}+a\left(x-\alpha^{\prime}\right)+\left[a\left(y-\beta^{\prime}\right)-\left(x-\alpha^{\prime}\right] \frac{\partial \beta}{\partial \alpha}\right] \partial \alpha=0 .\right.
\end{aligned}
$$

Setzt man nun hierin für $y-\beta$ und $y-\beta^{\prime}$ die Werthe aus (1. und 2.), so verschwinden, in Folge der Gleichung (3.), die Coëfficienten von $\partial \alpha$ und $\partial \alpha^{\prime}$. Der Ausdruck für den Differential-Coëfficienten $\frac{\partial y}{\partial x}$ ist daher 
derselbe; man mag $\alpha, \alpha^{\prime}$ als veriinderlich oder als constant betrachten. Hieraus folgt, dals die Tangente und die Normale des Ortes, den der Punct $x, y$ beschreibt, mit der Tangente und Normale des Kreises (4.) zusammenfallen; und man hat den Satz:

1) Wenn ein Winkel von veränderlicher Grörse sich so bewegt, dafs seine Schenkel fortwährend eine gegebene ebene Curve berühren, so beschreibt die Spitze $P$ dieses Winkels eine ebene Cure von der Beschaffenheit, dafs die Normale in einem Puncte $P$ dieser Curve durch den Mittelpunct des Kreises geht, welcher durch den Punct $P$ und die Puncte $A, B$, in denen die Schenkel des Winkels die gegebene Curve berühren, gelegt werden kann.

Es ist klar, dafs dieser Satz auch noch Statt findet, wenn der Winkel sich so bewegt, dafs der eine Schenkel eine und der andere Schenkel eine andere Curve berührt.

Lälst man zwei gerade Linien sich so bewegen, dafs sie fortwährend Normalen an einer gegebenen Curve sind und mit einander denselben constanten Winkel bilden, so werden sie zugleich die Evolute jener Curve berühren, und man kann daher folgenden Satz aufstellen:

2) Wenn ein Schenkel von unveränderlicher Gröfse sich in einer Ebene so bewegt, dals seine Schenkel eine gegebene, in derselben Ebene liegende Curve rechtwinklig schneiden, so beschreibt die Spitze $P$ dieses Winkels eine Curve von der Beschaffenheit, dals die Normale in einem ihrer Puncte $P$, durch den Mittelpunct des Kreises geht, welcher durch den Punct $P$ und die Krümmungs-Mittelpuncte $A^{\prime}$, $B^{\prime}$ der Puncte $A$ und $B$, in denen die Schenkel des Winkels die gegebene Curve schneiden, gelegt werden kann.

Obgleich diese Sätze von grofser Allgemeinheit sind, so lassen sie sich aus noch allgemeineren als besondere Fälle ableiten. Wenn nemlich zwei gerade Linien sich so bewegen, dafs sie fortwährend eine gegebene ebene Curve berühren, und dafs sie mit einer dritten geraden Linie von gegebener Lage zwei Winkel bilden, die auf irgend eine gegebene Art von einander abhangen, so wird der Durchschnittspunct jener beiden Linien eine Curve beschreiben, deren Gestalt von derjenigen der gegebenen Curve und von der gegebenen Art der Abhängigkeit der genanten 
beiden Winkel bestimmt werden wird. Man nehme nun die gerade Linie von gegebener Lage zur Abscissen-Achse und nenne die oben durch $\frac{\partial \beta}{\partial \alpha}, \frac{\partial \beta^{\prime}}{\partial \alpha^{\prime}}$ bezeichneten Differential-Coëfficienten, der Kürze wegen, $p$ und $p^{\prime}$, so sind die Gleichungen der beiden beweglichen Tangenten

$$
\begin{aligned}
\text { I. } y-\beta=p(x-\alpha) \text {, } \\
\text { II. } y-\beta^{\prime}=p^{\prime}\left(x-\alpha^{\prime}\right) \text {. }
\end{aligned}
$$

Die Abhängigkeit der Winkel, welche diese beiden Linien mit der $\mathbf{A b}$ scissen-Achse bilden, wird sich immer durch eine Gleichung zwischen den Tangenten dieser Winkel, d. i. zwischen $p$ und $p^{\prime}$, angeben lassen, so dafs man hat:

$$
\text { III. } \psi\left(p, p^{\prime}\right)=0 \text {, }
$$

wo $\psi$ eine gegebene Function bedeutet.

Setzt man nun für $p$ und $p^{\prime}$ die Werthe aus (I., II.) in (III.), so kommt

$$
\text { IV. } \psi\left(\frac{y-\beta}{x-\alpha}, \frac{y-\beta^{\prime}}{x-\alpha^{\prime}}\right)=0 \text {, }
$$

und diese Gleichung drückt, wenn man $\alpha, \beta$ und $\alpha^{\prime}, \beta^{\prime}$ als constant ansieht, diejenige Curve aus, die der Durchschnittspunct zweier geraden Linien beschreiben würde, wenn diese letzteren sich um die als fest betrachteten Puncte drehen, während sie mit der Abscissen-Achse Winkel von der gegebenen Abhängigkeit bilden.

Differentiirt man die Gleichung (IV.), indem man, aufser $y$ und $x$, auch $\alpha \beta$ und $\alpha^{\prime} \beta^{\prime}$ als veränderlich betrachtet, so kommt

$$
\begin{gathered}
\text { V. }\left(\frac{\partial \psi}{\partial p} \cdot \frac{1}{x-\alpha}+\frac{\partial \psi}{\partial p^{\prime}} \cdot \frac{1}{x-\alpha^{\prime}}\right) \partial y-\left(\frac{\partial \psi}{\partial p} \cdot \frac{y-\beta}{(x-\alpha)^{2}}+\frac{\partial \psi}{\partial p^{\prime}} \cdot \frac{y-\beta^{\prime}}{\left(x-\alpha^{\prime}\right)^{2}}\right) \\
-\frac{\partial \psi}{\partial p}\left(\frac{(x-\alpha) \frac{\partial \beta}{\partial \alpha}-(y-\beta)}{(x-\alpha)^{2}}\right) \partial \alpha-\frac{\partial \psi}{\partial p^{\prime}}\left(\frac{\left(x-\alpha^{\prime}\right) \frac{\partial \beta^{\prime}}{\partial \alpha^{\prime}}-\left(y-\beta^{\prime}\right)}{\left(x-\alpha^{\prime}\right)^{2}}\right) \partial \alpha^{\prime}=0 .
\end{gathered}
$$

In dieser Gleichung verschwinden aber, in Folge der Gleichungen (I., II.), die Coëfficienten von $\partial \alpha$ und $\partial \alpha^{\prime}$, d. i. der Ausdruck für den Differential-Coëfficienten $\frac{\partial y}{\partial x}$ ist derselbe, man mag $\alpha \beta$ und $\alpha^{\prime} \beta^{\prime}$ als verïnderlich oder als constant betrachten, und hieraus ergiebt sich der Satz:

3) Wenn zwei Tangenten an einer gegebenen Curve sich so bewegen, dafs sie mit einer-festen Linie Winkel bilden, die auf irgend eine gegebene Weise von einander abhangen, so beschreibt der Durchschnitspunct $P$ dieser Tangenten eine Curve von der Beschaffenheit, dals ihre Berührungslinie im Puncte $P$ mit der Berührungslinie derjenigen 
Curve zusammenfält, in welcher der Punct $P$ liegen würde, wenn die beiden beweglichen Tangenten, statt sich an der gegebenen Curve fortzuschieben, sich um die, dem Puncte $P$ entsprechenden Berührungspuncte der gegebenen Curve als feste Puncte drehten.

Aus diesem Satze lïlst sich durch Betrachtung der Evolute der folgende herleiten:

4) Wenn zwei Normalen an einer gegebenen Curve sich so bewegen, dafs sie mit einer festen Linie Winkel bilden, die auf irgend eine gegebene Weise von einander abhangen, so beschreibt der Durchschnitspunct $P$ dieser Normalen eine Curve von der Beschaffenheit, dafs ihre Berührunglinie im Puncte $P$ mit der Berühunglinie derjenigen Curre zusammenfält, in welcher der Punct $P$ liegen würde, wenn die beiden beweglichen Normalen, statt sich auf der gegebenen Curve fortzuschieben, sich um die Krümungs-Mittelpuncte derjenigen Puncte drehten, in welchen die Normalen durch den Punct $P$ die gegebene Curve schneiden.

Es scien $y=\varphi(x) ; y=\varphi_{1}(x) ; \ldots . y=\varphi_{n}(x)$ die Gleichungen einer beliebigen Anzahl gegebener Curven, und von einem Puncte $P$ der Ebene, welche diese Curven enthält, sei an jeder derselben eine Normale gezogen. Wenn nun die Längen dieser Normalen, vom Puncte $P$ bis zu den Curven genommen, in einer nur durch eine einzige Gleichung ausgedrückten Relation stehen, so wird der Punct $P$ eine Curve beschreiben. Nennt man die Coordinaten des Punctes $P x$ und $y$; diejenigen der Puncte $A$, $A^{\prime}, \ldots . A^{(n)}$, in welchen die genannten Curven von den Normalen geschnitten werden, $\alpha \beta, \alpha^{\prime} \beta^{\prime}, \ldots \alpha^{(n)} \beta^{(n)}$; und $u, u_{3}, \ldots u_{n}$ die Längen dieser Normalen, so hat man, aufser den Gleichungen $\beta=\varphi(\alpha), \beta^{\prime}=\varphi_{1}(\alpha), \ldots$ ... $\beta^{(n)}=\varphi_{n}\left(\alpha^{(n)}\right)$, zur Bestimmung des Ortes von $P$ die Gleichungen: (a.) $(y-\beta) p+(x-\alpha)=0 ;\left(y-\beta^{\prime}\right) p^{\prime}+\left(x-\alpha^{\prime}\right)=0 ; \ldots\left(y-\beta^{(n)}\right) p^{(n)}+\left(x-\alpha^{(n)}\right)=0$, (b.) $(y-\beta)^{2}+(x-\alpha)^{2}=u^{2} ;\left(y-\beta^{\prime}\right)^{2}+\left(x-\alpha^{\prime}\right)^{2}=u_{x}^{2} ; \ldots .\left(y-\beta^{(n)}\right)^{2}+\left(x-\alpha^{(n)}\right)^{2}=u_{n}^{2}$, (c.) $\psi\left(u, u_{1}, \ldots u_{n}\right)=0$,

wo $p, p^{\prime}, \ldots \ldots p^{(n)}$ für $\frac{\partial \beta}{\partial \alpha} ; \frac{\partial \beta^{\prime}}{\partial \alpha^{\prime}} ; \ldots \frac{\partial \beta^{(n)}}{\partial \alpha^{(n)}}$ gesetzt sind, und $\psi$ eine gegebene Function bedeutet. Entwickelt man nemlich aus den zuerst ge- 
nannten Gleichungen die Grölsen $\alpha, \beta, \alpha^{\prime}, \beta^{\prime}, \ldots \alpha^{(n)}, \beta^{(n)}$, und setzt sie in die letzte Gleichung:

$$
\psi\left(u, u_{1}, \ldots . u_{n}\right)=0
$$

so erhält man die Gleichung des Ortes von $P$ in $x$ und $y$ ausgedrückt. Wenn man aber diese Gleichung differentiirt, so kommt:

$$
\text { (d.) }\left\{\begin{array}{c}
{\left[\frac{\partial \psi}{\partial u} \cdot \frac{\partial u}{\partial y}+\frac{\partial \psi}{\partial u_{x}} \cdot \frac{\partial u_{x}}{\partial y} \ldots+\frac{\partial \psi}{\partial u_{n}} \cdot \frac{\partial u_{n}}{\partial y}\right] \partial y} \\
+\left[\frac{\partial \psi}{\partial u} \cdot \frac{\partial u}{\partial x}+\frac{\partial \psi}{\partial u_{x}} \cdot \frac{\partial u_{x}}{\partial x} \ldots+\frac{\partial \psi}{\partial u_{n}} \cdot \frac{\partial u_{n}}{d x}\right] \partial x \\
+\frac{\partial \psi}{\partial u} \cdot \frac{\partial u}{\partial \alpha} \partial \alpha+\frac{\partial \psi}{\partial u_{x}} \cdot \frac{\partial u_{x}}{\partial \alpha^{\prime}} \partial \alpha^{\prime} \ldots+\frac{\partial \psi}{\partial u_{x}} \cdot \frac{d u_{n}}{\partial \alpha^{(n)}} \partial \alpha^{(n)}=0 .
\end{array}\right.
$$

Es ist aber auch:

$$
\begin{gathered}
\frac{\partial u}{\partial \alpha}=-\frac{1}{\mu}\left[(y-\beta) \frac{\partial \beta}{\partial \alpha}+(x-\alpha)\right] ; \frac{\partial u_{x}}{\partial \alpha^{\prime}}=-\frac{1}{u_{x}}\left[(y-\beta) \frac{\partial \beta^{\prime}}{\partial \alpha^{\prime}}+\left(x-\alpha^{\prime}\right)\right] ; \\
\ldots \frac{\partial u_{n}}{\partial \alpha^{(n)}}=-\frac{1}{u_{n}}\left[\left(y-\beta^{(n)}\right) \frac{\partial \beta^{(n)}}{\partial \alpha^{(n)}}+\left(x-\alpha^{(n)}\right)\right],
\end{gathered}
$$

also in Folge der Gleichungen $\left(a_{0}\right)$ :

$$
\frac{\partial u}{\partial \alpha}=0 ; \quad \frac{\partial u_{x}}{\partial \alpha^{\prime}}=0 ; \ldots . \frac{\partial u_{n}}{\partial \alpha^{(n)}}=0 ;
$$

und es verschwinden demnach in der Gleichung $\left(d_{\text {. }}\right)$ die Coëfficienten von $\partial \alpha, \partial \alpha^{\prime}, \ldots \partial \partial \alpha^{(n)}$, so dafs der Ausdruck von $\frac{\partial y}{\partial x}$ derselbe ist, man mag $\alpha \beta, \alpha^{\prime} \beta, \ldots$. als veränderlich oder als constant betrachten. Wenn aber diese letztern Gröfsen als constant angesehen werden, drückt die Gleichung $\psi\left(u, u, \ldots \ldots u_{n}\right)=0$ den Ort des Punctes $P$, für den Fall dals $A$, $A^{\prime}, \ldots \ldots A^{(n)}$ feste Puncte sind, aus. Die Tangente im Puncte $P$ des erstgenannten Ortes fällt demnach mit der Tangente desselben Punctes $P$ im letztgenannten zusammen, und man kann daher den folgenden Satz aufstellen :

5) Wenn ein Punct $P$ sich in einer Ebene so bewegt, dafs die Länge der Normalen, die von diesem Puncte an eine beliebige Anzahl gegebener, in derselben Ebene liegender Curven gezogen werden können, in einer bestimmten durch eine einzige Gleichung augedrückten Relation stehen, so ist der Ort des Punctes $P$ so beschaffen, dafs die Tangente im Puncte $P$ mit der Tangente derjenigen Curve zusammenfällt, in welcher der Punct $P$ liegen würde, wenn die beweglichen Normalen statt sich auf den gegebenen Curven fortzuschieben, sich um die dem Puncte $P$ entsprechenden Durchschnittspuncte $A, A^{\prime}, \ldots A^{(n)}$ drehten. 
Um aus diesem allgemeinen Satze einige andere herzuleiten, wollen wir annehmen, der Punct $P$ soll so liegen, dafs die Summe der Quadrate der Normalen eine gegebene constante Gröfse sei. Nach einem bekannten Satze ist nun der Ort des Punctes $P$, wenn die Puncte $A, A^{\prime}, \ldots$ $\ldots A^{(n)}$ fest sind, ein Kreis, dessen Mittelpunct der Schwerpunct von $A$, $A^{\prime}, \ldots A^{(n)}$ ist, und hieraus haben wir nun unmittelbar den folgenden:

6) Wenn ein Punct $P$ in einer Ebene sich so bewegt, dafs die Summe derQuadrate der Normalen, welche von diesem Puncte an eine oder mehrere Curven in derselben Ebene gezogen werden können, einer gegebenen constanten Gröfse gleich ist, so geht die Normale des Ortes von $P$ durch den Schwerpunct derjenigen Puncte, in welchen die vom Puncte $P$ an die gegebenen Curven gezogenen Normalen diese Curven schneiden.

Wir wollen ferner annehmen, die Summe (oder die Differenz) zweier Normalen, welche vom Puncte $P$ aus an eine oder zwei gegebene Curven gezogen werden, sei constant. In diesem Falle ist der Ort des Punctes $P$, für constante Puncte $A, A^{\prime}$, eine Ellipse (oder Hyperbel) und $A, A^{\prime}$ ihre Brempuncte, Daher:

7) Wenn ein Punct $P$ sich in einer Ebene so bewegt, dafs die von diesem Puncte aus an eine oder zwei gegebene Curven gezogenen Normalen $P A, P A^{\prime}$ eine constante Summe (oder Differenz) bilden, so halbirt die Normale (oder Tangente) des Ortes ron $P$ den Winkel $A P A^{\prime}$, welchen die beiden Normalen einschliefsen.

\section{3.}

Wenn nicht die Längen der Normalen, sondern die Längen der vom Puncte $P$ aus an die Curven gezogenen Tangenten in einer bestimmten Relation stehen sollen, so ist die Lage der Berührungslinie im Puncte $P$ nicht mehr von der Krümmung der gegebenen Curven in den Puneten $A, A^{\prime}, \ldots, A^{(n)}$ unabhängig. Denn statt der Gleichungen (a.) hat man jetzt die Gleichungen:

( $\left.a^{\prime}.\right) \quad(y-\beta)-p(x-\alpha)=0 ;\left(y-\beta^{\prime}\right)-p^{\prime}\left(x-a^{\prime}\right)=0 ; \ldots .\left(y-\beta^{(n)}\right)-p^{(n)}\left(x-\alpha^{(n)}\right)=0$, und in Folge dieser Gleichungen sind die Coëfficienten von $\partial \alpha, \partial \alpha^{\prime}, \ldots$ $\ldots \partial \alpha^{(n)}$ in der Gleichung $\left(d_{\text {o }}\right)$ nicht gleich Null. Setzt man aber für $\frac{\partial u}{\partial y}$ und $\frac{\partial u}{\partial x}$ die sich aus $(b$,$) ergebenden Werthe \frac{1}{u_{0}}(y-\beta)$ und $\frac{1}{u_{0}}(x-a)$; Crelle's Jinarnal d. M. VAL, Bd. 2. Hft. 
fiir $\frac{\partial u_{x}}{\partial y}, \ldots \frac{\partial u_{n}}{\partial y}$, und für $\frac{\partial u_{x}}{\partial x}, \ldots \frac{\partial u_{n}}{\partial x}$ die analogen; ferner für $\partial \alpha$, $\partial \alpha^{\prime}, \ldots \partial \alpha^{(n)}$ die sich aus $\left(a^{\prime}.\right)$ ergebenden $\frac{\partial y-p \partial x}{(x-a) q} ; \frac{\partial y-p^{\prime} \partial x}{\left(x-\alpha^{\prime}\right) q^{\prime}}$ etc., wo $q, q^{\prime}$ die Differential-Coëfficienten $\frac{\partial^{2} \beta}{\partial \alpha^{2}} ; \frac{\partial^{2} \beta^{\prime}}{\partial \alpha^{\prime 2}}$ etc. bezeichnen, so erhïlt man aus $(d$.

(d $d^{\prime}$ ) $\left\{\frac{\partial \psi}{\partial u} \cdot \frac{1}{u}\left[y-\left(\beta+\frac{1+p^{2}}{q}\right)\right]+\frac{\partial \psi}{\partial u_{\mathrm{x}}} \cdot \frac{1}{u_{\mathrm{x}}}\left[y-\left(\beta^{\prime}+\frac{1+p^{\prime 2}}{q^{\prime}}\right)\right]+\ldots ..\right\} \rho y$ $+\left\{\frac{\partial \psi}{\partial u} \cdot \frac{1}{u}\left[x-\left(\alpha-\frac{\left(1+p^{2}\right) p}{q}\right)\right]+\frac{\partial \psi}{\partial u_{x}} \cdot \frac{1}{u_{x}}\left[x-\left(\alpha^{\prime}-\frac{\left(1+p^{\prime 2}\right) p^{\prime}}{q^{\prime}}\right)\right]+\ldots.\right\} \partial x=0$, oder, wenn man $\beta+\frac{1+p^{2}}{q}, \beta^{\prime}+\frac{1+p^{\prime 2}}{q^{\prime}}$ etc.; $\alpha-\frac{\left(1+p^{2}\right) p}{q}, \alpha^{\prime}-\frac{\left(1+p^{\prime 2}\right) p^{\prime}}{q^{\prime}}$, welche nichts anders als die Coordinaten der Krümmungs - Mittelpuncte von $A, A^{\prime}$ etc. sind, durch $\delta, \delta^{\prime}$ etc.; $\gamma, \gamma^{\prime}$ etc. bezeichnet:

$\left[\frac{\partial \psi}{\partial u} \cdot \frac{1}{u}(y-\delta)+\frac{\partial \psi}{\partial u_{x}} \cdot \frac{1}{u_{x}}\left(y-\delta^{\prime}\right)+\ldots.\right] \partial y+\left[\frac{\partial \psi}{\partial u} \cdot \frac{1}{u}(x-\gamma)+\frac{\partial \psi}{\partial u_{x}} \cdot \frac{1}{u_{x}}\left(x-\gamma^{\prime}\right)+\ldots.\right]=0$.

Ist z. B. die Summe der Quadrate der Tangenten eine constante Grölse, so dafs man hat

$$
\psi\left(u, u_{x}, \ldots . u_{n}\right)=u^{2}+u_{1}^{2}+\ldots . u_{n}^{2}-c^{2}=0,
$$

so ist

$$
\frac{\partial \psi}{\partial u} \cdot \frac{1}{u}=\frac{\partial \psi}{\partial u_{x}} \cdot \frac{1}{u_{x}}=\ldots .=\frac{\partial \psi}{\partial u_{n}} \cdot \frac{1}{u_{n}}=2,
$$

und für diesen Fall entsteht aus der obigen Gleichung:

$$
\left[y-\frac{\delta+\delta^{\prime}+\ldots .+\delta^{(n)}}{n+1}\right] \partial y+\left[x-\frac{\gamma+\gamma^{\prime}+\ldots .+\gamma^{(n)}}{n+1}\right] \partial x=0 .
$$

Die Gleichung der Normale im Puncte $x y$ des Ortes von $P$ ist daher

$$
Y-y=\frac{y-\frac{\delta+\delta^{\prime}+\ldots .+\delta^{(n)}}{n+1}}{x-\frac{\gamma+\gamma^{\prime}+\ldots+\gamma^{(n)}}{n+1}}(X-x),
$$

wenn die laufenden Coordinaten dieser Normale durch $X, Y$ bezeichnet werden. Hieraus ist klar, dafs die Normale durch den Schwerpunct der Krümmungs-Mittelpuncte geht. Wir haben daher folgenden Satz:

8) Wenn ein Punct $P$ sich in einer Ebene so bewegt, dafs die Summe der Quadrate der Tangenten $P A, P A^{\prime}$ etc., welche von diesem Puncte an eine oder mehrere in derselben Ebene liegende Curven gezogen werden, constant ist, so geht die Normale des Ortes von $P$ durch den Schwerpunct der Krümmungs-Mittelpuncte von $A, A^{\prime}$ etc. 
Es seien ferner nur zwei Tangenten von $P$ aus gezogen und diese seien einander gleich, so hat man

daher

folglich

$$
\psi\left(u, u_{1}, \ldots . u_{n}\right)=u-u_{1}=0 \text {; }
$$

$$
\frac{\partial \psi}{\partial u} \cdot \frac{1}{u}=\frac{1}{u} \text { und } \frac{\partial \psi}{\partial u_{x}} \cdot \frac{1}{u_{x}}=-\frac{1}{u} \text {, }
$$

Die Gleichung der Normale ist also

$$
\left(\delta^{\prime}-\delta\right) \partial y+\left(\gamma^{\prime}-\gamma\right) \partial x=0 \text {. }
$$

$$
Y-y=\frac{\delta^{\prime}-\delta}{\gamma^{\prime}-\gamma}(X-x)
$$

und diese Linie mithin der Verbindungslinie der Krümmungs-Mittelpuncte parallel, oder

9) Der Ort der gleichen Tangente an einer oder zwei ebenen Curven ist so beschaffen, dafs dieTangente in einem Puncte $P$ desselben, auf der Verbindungslinie der Krümmungs-Mittelpuncte der zugehörigen Berührungspuncte an den gegebenen Curven senkrecht steht.

\section{4.}

Wir haben oben gesehen, dafs die Lage der Berührungslinie im Puncte $P$ unabhängig sei von der Krümmung der gegebenen Curven in den Puncten $A, A^{\prime}$ etc., es mag $\psi$ eine Form haben, welche man will, wenn nemlich für den Fall der von $P$ aus gezogenen Tangenten $p, p^{\prime}$ etc., oder was dasselbe ist, $\frac{y-\beta}{x-\alpha}, \frac{y-\beta^{\prime}}{x-\alpha^{\prime}}$ etc., und für den Fall der von $P$ aus gezogenen Normalen, $\checkmark\left[(y-\beta)^{2}+(x-\alpha)^{2}\right] ; V\left[\left(y-\beta^{\prime}\right)^{2}+\left(x-\alpha^{\prime}\right)^{2}\right]$ etc. die Functionalgrölsen in $\psi$ sind. Es kann nun gefragt werden, welches die Functionalgrölsen in $\psi$ scin müssen, wenn vom Puncte $P$ aus gerarle Linien an die gegebenen Curven gezogen werden, die diese unter irgend einem bestimmten Winkel schneiden, damit die Lage der Berührungslinie in $P$ von den Krümmungen der gegebenen Curven in $A, A^{\prime}$ etc. unabhïngig seien. Eine leichte Rechnung ergiebt für die Form dieser Functionalgröfsen im Allgemeinen :

$$
\begin{aligned}
& e^{-\frac{2}{a} \operatorname{Arc}\left(\operatorname{tang}=\frac{y-\beta}{x-\alpha}\right)} \cdot V\left[(y-\beta)^{2}+(x-\alpha)^{2}\right], \text { oder } \\
& e^{-\operatorname{Arc}\left(\operatorname{tang}=\frac{y-\beta}{x-\alpha}\right)} \cdot\left[(y-\beta)^{2}+(x-\alpha)^{2}\right]^{\frac{\alpha}{2}},
\end{aligned}
$$

wo $a$ die trigonometrische Tangente des Winkels bedeutet, unter welchem die gegebenen Curven von den beweglichen geraden Linien geschnitten werden, und wo $e$ die Basis der natürlichen Logarithmen bezeichnet. 